\title{
Protective Effects of Grape Seed Proanthocyanidin Extracts on Cerebral Cortex of Streptozotocin-Induced Diabetic Rats through Modulating AGEs/RAGE/NF-kB Pathway
}

\author{
Mei $\mathrm{Lu}^{1,2}$, Ling Xu ${ }^{1,2}$, Baoying $\mathrm{LI}^{1,2}$, Weidong ZHANG ${ }^{3}$, Chengmei ZHANG ${ }^{4}$, \\ Hong $\mathrm{FeNG}^{1,2}$, Xiaopei $\mathrm{CuI}^{1,2}$ and Haiqing $\mathrm{GAO}^{1,2, *}$ \\ ${ }^{1}$ Key Laboratory of Cardiovascular Proteomics of Shandong Province, Jinan, China \\ ${ }^{2}$ Department of Geriatrics, QiLu Hospital, Shandong University, Jinan, China \\ ${ }^{3}$ Department of Pathology, Institute of Basic Science, Medical Science Academy of Shandong, Jinan, China \\ ${ }^{4}$ Division of Transgenic Animal Science, Shandong University, Jinan, China
}

(Received September 9, 2009)

\begin{abstract}
Summary Diabetic encephalopathy is a severe complication in patients with long-term hyperglycemia. Oxidative stress is thought to be closely implicated in this disorder, so in this study, we examined whether grape seed proanthocyanidin extract (GSPE), a naturally occurring antioxidant derived from grape seeds, could reduce the injuries in the cerebral cortex of diabetic rats by modulating advanced glycation end products (AGEs)/the receptor for AGEs (RAGE)/nuclear factor-kappa B p65 (NF- $\kappa$ B p65) pathway, which is crucial in oxidative stress. Body weight and serum AGEs were tested; cerebral cortexes were isolated for morphological observations and the pyramidal cell layers were immunohistochemically stained for the detection of RAGE, NF- $\kappa$ B p65, intercellular adhesion molecule-1 (ICAM-1) and vascular cell adhesion molecule-1 (VCAM-1) as well. For RAGE and NF- $\kappa$ B p65, quantitative reverse transcriptase coupled to polymerase chain reaction (RT-PCR) was employed for determination of mRNA levels, and western blot was used to detect protein expression. Our results showed that long term hyperglycemia in diabetic rats caused the degeneration of neurons and the up-regulation of serum AGEs, and also the up-regulation of RAGE, NF- $\kappa B$ p65, VCAM-1 and ICAM-1 in the brain. We found that GSPE treatment improved the pathological changes of diabetic rats by modulating the AGEs/RAGE/NF- $\kappa \mathrm{B}$ p65 pathway. This study enables us to further understand the key role that the AGEs/RAGE/NF- $\kappa \mathrm{B}$ pathway plays in the pathogenesis of diabetic encephalopathy, and confirms that GSPE might be a therapeutical means to the prevention and treatment of this disorder.
\end{abstract}

Key Words diabetic encephalopathy, grape seed proanthocyanidin extracts, advanced glycation end products, nuclear factor-kappa B p65, cerebral cortex

Patients with diabetes have at least twice the risk of stroke and may show performance deficits in a wide range of cognitive domains varying from mild to moderate, which significantly impair their daily quality of life (1-3). Although the microvascular complications of diabetes have been well described, the concept of central neuropathy, which was first introduced in 1950 as diabetic encephalopathy, has remained controversial for more than 80 y since it was first described in 1922 (4). To date, diabetic encephalopathy still needs a clear definition and assessment criteria. Recent studies reveal that the mechanisms underlying this most hazardous disorder involve not only vascular changes but also direct damage to neurons induced by superoxide overproduction and subsequent inflammatory cataract $(2$, $5,6)$.

Advanced glycation end products, i.e. AGEs, the final products of nonenzymatic glycation and oxidation of proteins and lipids, were found to accumulate in

*To whom correspondence should be addressed.

E-mail: gaohaiqing52@yahoo.com.cn human tissues with aging and at an accelerated rate in diabetes (2, 5-7). The receptor for AGEs (RAGE), an integral membrane protein, forming the central binding site on the cell surface for AGEs, is a multiligand and signal transduction receptor belonging to the immunoglobulin superfamily and can be up-regulated under various pathological conditions $(5,7,8)$. The engagement of AGEs to RAGE leads to the activation of the transcription factor nuclear factor kappa $\mathrm{B}(\mathrm{NF}-\kappa \mathrm{B})$ and subsequent expression of NF- $\kappa \mathrm{B}-$ regulated cytokines, including adhesion molecules such as vascular cell adhesion molecule-1 (VCAM) and intercellular adhesion molecule-1 (ICAM-1) (9). A wide range of chronic disorders were propagated according to a two-hit model, with the increased expression of RAGE itself ( 5 , 8 ). The literature shows that the AGEs/RAGE/NF- $\kappa \mathrm{B}$ pathway plays a vital role in proinflammatory responses, accelerated diabetic atherosclerosis, Alzheimer's disease, and tumor cell invasion $(7,10)$. So it is reasonable to conclude that the AGEs/RAGE/NF- $\kappa$ B pathway may be an important factor in the progression of various neurodegenerative disorders, including dia- 
betic encephalopathy $(8,10-12)$. In the present study, we try to find interventions to antagonize this pathway, which may lead to a therapy for diabetic encephalopathy.

The phenomenon that red wine can reduce the mortality and morbidity of coronary vascular diseases has attracted considerable attention for a long time (10, 13-19). Polyanthocyanidins, which are oligomers and polymers of polyhydroxy flavan-3-ol units, such as (+)catechin and (-)-epicatechin, have been found to be the major polyphenols in red wine and grape seeds (13, $14,16,18)$. The pharmacological activity and therapeutical effects of grape seed proanthocyanidin extract (GSPE) include reducing the incidence of ischemia-reperfusion induced arrhythmia in the isolated rat heart, performing cardiomyocyte protection against exogenous $\mathrm{H}_{2} \mathrm{O}_{2}$-induced as well as endogenous antimycin Ainduced oxidant stress $(12-15,17)$. GSPE also functions as a reactive oxygen and nitrogen species scavenger, immune function modulator (17), antithrombotic agent (15), and LDL oxidation inhibitor (14, 16). Besides, GSPE has demonstrated hypoglycemic effect in STZ-induced diabetic rats (20), which is our biggest concern in this experiment.

Our previous study demonstrated that GSPE could inhibit the enhanced VCAM-1 expression in AGEexposed endothelial cells by suppressing reactive oxygen species (ROS) generation (21). Most important of all, our latest systemic research found that GSPE significantly decreases the mRNA and protein expression levels of RAGE and NF- $\kappa$ B p 65 in the hippocampus of diabetic rats (22). No evidence so far has been found of toxic effects, mutagenic potential, or teratogenic effects of an appropriate amount of GSPE, which means that the safety of GSPE treatment is devoid of worry (13). Thus, GSPE may be used as an effective intervention in chronic diabetic complications.

To further understand the roles that the AGEs/RAGE/ $\mathrm{NF}-\kappa \mathrm{B}$ pathway plays in the pathogenesis of diabetic encephalopathy, in this study, we examined whether GSPE might modulate the expression of RAGE, NF- $\kappa \mathrm{B}$ p65 and the downstream targets VCAM-1 and ICAM-1 in the pyramidal cell layers of the cerebral cortex in STZ-induced diabetic rats. We hypothesized that RAGE was a key molecular target for preventional therapy in diabetic encephalopathy.

\section{MATERIALS AND METHODS}

Experimental animal model. Sixty male Wistar rats weighing 180-220 g were purchased from the Animal Centre of Shandong University. Animals were kept in individual cages on a 12-h light-dark cycle with an ambient temperature of $22 \pm 1^{\circ} \mathrm{C}$ and free access to food and water. The care of the animals and research protocols conformed to the guiding principles for animal experimentation as expounded by the Chinese Council on Animal Care and approved by the Animal Care Committee of Shandong University.

Grouping and intervention. Twenty rats selected randomly were divided into 2 groups: control group 1 (C1, $n=10)$ without GSPE treatment and control group 2 $(\mathrm{C} 2, n=10)$ with GSPE treatment. The other forty rats received a single dosage of pancreatic beta cell toxin streptozotocin (Sigma, St. Louis, MO, USA) $55 \mathrm{mg} / \mathrm{kg}$ body weight, which was injected into tail veins of the rats freshly dissolved in $0.1 \mathrm{M}$ sodium citrate buffer $(\mathrm{pH}$ 4.5 ) after a $12 \mathrm{~h}$ overnight fasting, while control animals received a single tail vein injection of 0.1 M sodium citrate buffer only. Glucose concentration from fasting animals was measured daily in a blood sample obtained from the tail by a pinprick, with a glucose oxidaseimpregnated test strip and a reflectance meter (One Touch $^{\circledR}$, Lifescan, USA). Only rats with blood sugar higher than $16.7 \mathrm{~mm}$ after $1 \mathrm{wk}$ were considered as diabetic. Ten rats with blood sugar levels less than $16.7 \mathrm{~mm}$ were excluded from the investigation. After 1 wk stabilization, the diabetic rats were divided randomly into 2 groups: one diabetic group without any hypoglycemic treatment (DM1, $n=15$ ) and the other diabetic group treated with GSPE with a daily dosage of $250 \mathrm{mg} / \mathrm{kg}$ body weight (DM2, $n=15$ ). Each group was observed from week 1 to week 24 without any hypoglycemic therapy. At the end of the research, there were $10,10,8,12$ rats left in group C1, C2, DM1, DM2, respectively. So, the grouping was as follows: control groups: $\mathrm{C} 1, n=10$, without GSPE treatment; $\mathrm{C} 2, n=10$, with GSPE treatment; diabetic groups: DM1, $n=8$, without GSPE treatment; DM2, $n=12$, with GSPE treatment.

GSPE treatment of diabetic rats. The interventional study with GSPE was conducted $1 \mathrm{wk}$ after diabetic induction. GSPE ( $56 \%$ dimeric proanthocyanidins, $12 \%$ trimeric proanthocyanidins, $6.6 \%$ tetrameric proanthocyanidins, small amounts of monomeric, high-molecular-weight oligomeric proanthocyanidins and flavonols (Lot No: G050412) were provided by Jianfeng, Inc. (Tianjin, China). The components of GSPE were analyzed by high-performance liquid chromatography (HPLC) with gas chromatography mass spectrometry detection. GSPE stock solutions for treatment were prepared fresh in phosphate buffered saline, i.e. PBS ( $137 \mathrm{mM} \mathrm{NaCl}, 3 \mathrm{mM} \mathrm{KCl}, 6 \mathrm{~mm} \mathrm{Na} 2 \mathrm{HPO}_{4} \cdot 12 \mathrm{H}_{2} \mathrm{O}, 2 \mathrm{mM}$ $\mathrm{KH}_{2} \mathrm{PO}_{4}, \mathrm{pH}$ 7.4), and were injected into the stomach of the rats in groups C2 and DM2 daily via an oro-gastric cannula attached to an external syringe, while rats in control groups (C1 and DM1) were given normal saline the same way. A weekly detection showed that plasma GSPE concentrations were $0.10-0.15 \mathrm{IU} / \mathrm{mL}$ in the GSPE treated rats.

Weight and parameters in blood. Animals were weighed weekly. Samples of blood from the tail were obtained for measurement of fasting blood glucose (FBG). When the rats were sacrificed, samples of intracardiac blood were obtained for biochemical, glycolated hemoglobin $\left(\mathrm{HbA}_{1 \mathrm{c}}\right)$ and AGE measurements. HbA $\mathrm{H}_{1 \mathrm{c}}$ was measured using high-performance liquid chromatography (DCA $2000^{+}$, Bayer, Germany). Other biochemical parameters such as lipid profile (TC, HDL-C, LDL-C and TG), creatinine and liver function were measured by automated enzymatic chemistry (HITACHI 
Table 1. Sequences of primer pairs used for real time quantitative RT-PCR.

\begin{tabular}{|c|c|c|c|}
\hline Name & $\begin{array}{c}\text { GenBank } \\
\text { accession number }\end{array}$ & Primer pairs & Product size (bp) \\
\hline RAGE & NM053336.1 & $\begin{array}{l}\text { Forward: 5'-actaccgagtccgagtctacca-3' } \\
\text { Reverse: } 5^{\prime} \text {-gctctgaccgaagcgtga-3' }\end{array}$ & $240 \mathrm{bp}$ \\
\hline NF- $\kappa$ B p65 & NM199267 & $\begin{array}{l}\text { Forward: } 5^{\prime} \text {-caccaaagacccacctcacc-3' } \\
\text { Reverse: } 5^{\prime} \text {-ggaccgcattcaagtcatagtc- } 3^{\prime}\end{array}$ & 220 bp \\
\hline GAPDH & X02231 & $\begin{array}{l}\text { Forward: } 5^{\prime} \text {-gaggggccacagtcttctg-3' } \\
\text { Reverse: } 5^{\prime} \text {-ccttcattgacctcaactacatggt- } 3^{\prime}\end{array}$ & 470 bp \\
\hline
\end{tabular}

7170 A, Japan). The serum AGE concentrations were detected by fluorescence spectrophotometer (HITACHI 850, Japan).

Tissue preparation. At the end of the intervention, the rats alive were sacrificed. Euthanasia for animals was performed with a dose of pentobarbital $(50 \mathrm{mg} / \mathrm{kg}$, i.p.) intraperitoneally perfused through the left ventricle for $10-15 \mathrm{~min}$ with a $0.9 \% \mathrm{NaCl}$ solution containing $0.5 \%$ polyvinypyrrolidone, heparin (20 international units), and ethylene diamine tatra acetic (EDTA, $25 \mathrm{mg} / \mathrm{kg}$ ) kept at $37^{\circ} \mathrm{C}$. This solution was replaced by a second one of $10 \%$ formalin in $0.1 \mathrm{M}$ PBS (pH 7.4), kept at room temperature. After $30 \mathrm{~min}$ of perfusion (perfusion pressure $1 \mathrm{~mL} / \mathrm{min}$ per $100 \mathrm{~g}$ body weight), the cerebral cortex was rapidly removed and frozen in powdered dry ice. In preparation for sectioning, brains were allowed to thaw sufficiently to cut the forebrain and brainstem into blocks using a razor blade. The cerebral cortex was selected. Tissue blocks were immediately embedded in Tissue-Tek (Miles) on a specimen holder and placed on the quick-freeze platform of an IEC Minotome at $-40^{\circ} \mathrm{C}$. Half of all cerebral cortex tissues were put in $2 \%$ formaldehyde and embedded in paraffin. For these specimens, $4 \mu \mathrm{m}$ sections were cut serially with a rotatory microtome for morphological and immunohistochemical studies. The other half of the cerebral cortex tissues were placed in Trizol reagent (Life Technologies Inc., Rockville Maryland, USA) and subsequently placed in liquid nitrogen for quantitative reverse transcriptase polymerase chain reaction (RT-PCR). Paraffin cerebral cortex sections were immunohistochemically stained for glial fibrillary acidic protein (GFAP), RAGE, NF- $\kappa$ B p65, VCAM-1 and ICAM-1.

Immunohistochemistry. Immunohistochemistry was performed on the paraffin-embedded tissue sections using a standard avidin-biotin complex-peroxidase method which uses 3,3'-diaminobenzidine as chromogen. Briefly, sections ( $4 \mu \mathrm{m}$ in thickness) were deparaffinized with xylene, and serial gradient concentrations of alcohol were used to dehydrate. Endogenous peroxidase was blocked by being incubated with 3\% hydrogen peroxide for $5 \mathrm{~min}$. After being washed with phosphate buffered saline, the sections were incubated in $10 \%$ sheep serum for $5 \mathrm{~min}$, followed by overnight incubation with rabbit anti-rat antibodies specific against soluble RAGE (sRAGE) (1:100, Dr. AM Schmidt, Columbia University, NY), NF- $\kappa$ B p65 (1 : 100, Biolegend, USA), GFAP (1:100 solution, Boster
Biotechnology Co., Wuhan, China), ICAM-1 and VCAM-1 (1 : 100 each, Boster Biotechnology Co.). Sections were then incubated with biotinylated antirabbit IgG followed by incubation in ABC Elite (HRP) reagent (Vector Laboratories). Reactions were visualized by developing in the DAB substrates (Vector Laboratories). Normal rabbit IgG of the same concentrations were used to replace the rabbit polyclonal antibodies as the negative control. All samples were visualized under a light microscope by an observer blinded to the brain conditions.

For immunohistochemistry comparison, immunostained neurons were analyzed from 4 randomly selected pyramidal cell layers of cerebral cortical regions. The numbers of GFAP positive neurons, the cell area of sRAGE, NF- $\kappa$ B p65, ICAM-1 and VCAM-1 positive neurons were determined and were compared between DM1 and C1, and DM1 and DM2. The gray values between white (0) and black (255) of positive neurons were recorded by Image Leica Qwin v3 Software (Leica DM 4000 B, Germany).

RNA extraction and quantitative RT-PCR. Total RNA was extracted from the brains using Trizol reagent (Life Technologies Inc., Rockville, MD). RNA was synthesized to cDNA using the TaqMan Reverse Transcription Reagents kit (Applied Biosystems). All PCR primers and TaqMan probes were designed using the software Primer Express (Applied Biosystems) and published sequence data from the NCBI database. Glyceraldehyde3-phosphate dehydrogenase (GAPDH) was designed as the housekeeping control gene. The sequences of primers are shown in Table 1.

Total RNA $(0.5 \mu \mathrm{g})$ was amplified using the Titan One Tube RT-PCR Kit (Boehringer-Mannheim, Shanghai, The People's Republic of China). The cycle profile was as follows: an initial denaturation at $95^{\circ} \mathrm{C}$ for $3 \mathrm{~min}$, followed by denaturation at $95^{\circ} \mathrm{C}$ for $30 \mathrm{~s}$, annealing at $60^{\circ} \mathrm{C}$ for $40 \mathrm{~s}$ and extension at $72^{\circ} \mathrm{C}$ for $30 \mathrm{~s}$ for NF- $\kappa \mathrm{B}$ p 65 and $1 \mathrm{~min}$ for RAGE, for 35 cycles, and a final extension at $72^{\circ} \mathrm{C}$ for $7 \mathrm{~min}$. The PCR products were separated by agarose gel electrophoresis and visualized by ethidium bromide staining. Bands were digitized by a Tanon-1000 Gel Image System (Shanghai, People's Republic of China). All reactions were performed in triplicate in an ABI PRISM 7900HT Sequence Detection System. Data were calculated and presented as the fold induction of mRNA for RAGE or NF- $\kappa$ B p 65 normalized to GAPDH, comparing the brain 
of diabetic rats to the brain of non-diabetic rats (defined as 1.0-fold).

Western blot. Western blot analysis was performed on samples of cerebral cortex obtained from the 4 groups of experimental rats. Tissues prepared were homogenized with ice-cold lysis buffer ( $10 \%$ glycerol, 2\% SDS, 25 mm Tris-HCl, pH 7.4, Roche Mini-Complete Protease Inhibitors), and then were centrifuged at $10,000 \times g$ for $15 \mathrm{~min}$. Supernatant was stored at $-20^{\circ} \mathrm{C}$ prior to immunoblotting analysis. The protein assay reagent for protein quantification was performed with the Bio-Rad Dc protein assay kit (Bio-Rad, Richmond, CA, USA). Equal amounts (100 $\mu \mathrm{g})$ of proteins were loaded and samples were separated by $12 \%$ SDSPAGE with $200 \mathrm{~V}$-h of current applied to a Mini-PROTEAN a2-D cell, Bio-Rad, USA. Separated proteins were transferred onto nitrocellulose membrane (BioRad) over $3 \mathrm{~h}$ at $200 \mathrm{~mA}$ in Towbin transfer buffer $(25 \mathrm{mM}$ Tris, $192 \mathrm{~mm}$ glycine, $20 \% \mathrm{v} / \mathrm{v}$ methanol, $0.1 \% \mathrm{v} / \mathrm{v}$ SDS). The blot was blocked for $1 \mathrm{~h}$ in $7.5 \%(\mathrm{w} / \mathrm{v})$ milk (Nestle, Carnation) in TBS (50 mm Tris, $137 \mathrm{~mm} \mathrm{NaCl}$, $51 \mathrm{~mm} \mathrm{KCl}$ ). Primary antibodies specific for sRAGE ( $35 \mathrm{kD}, 1: 400$, R\&D System, USA), activated NF- $\kappa$ B p65 $(1: 200$, Biolegend, USA) and $\beta$-actin $(1: 500$, AbCam, UK) were applied. Secondary anti-rabbit IgG HRP linked antibody (Cell Signaling Technology, Danvers, MA, USA) was applied at a $1: 7,500$ titer in each case. Signal detection was performed via exposing the blot to enhanced DAB color reagents (Zhong Shan Goldenbridge Biotechnology Company Limited, China) for $5 \mathrm{~min}$. The densointensity of RAGE or NF- $\kappa \mathrm{B}$ p65 was normalized to $\beta$-actin for each brain tissue. Luminosity quantification of each identified protein band was performed using Adobe Photoshop software (Adobe Photoshop 7.0, Adobe, San Jose, CA, 2002).

Statistical analyses. Data collected from the investigation groups were expressed as mean \pm standard deviation (SD). One-way ANOVA was used to compare means among all the groups at the end of the study. Student's $t$ test was employed to compare means between the diabetic group (DM1) and non-diabetic control group (C1), and means between the diabetic group (DM1) and GSPE-treated diabetic group (DM2). Results were considered significant at $p<0.05$. All analyses were performed with SPSS for Windows software version 13.0 (SPSS, Chicago, USA).

\section{RESULTS}

The rats were maintained in a diabetic state over the course of the study without any hypoglycemic treatment. The numbers remaining alive at the end of the study in the 4 groups $(\mathrm{Cl}$ : untreated non-diabetic group; C2: GSPE-treated non-diabetic group, DM1: untreated diabetic group; DM2: GSPE-treated diabetic group) were $10,10,8$ and 12 , respectively.

Weight and parameters in blood

As we have demonstrated before, diabetic rats (DM1 and DM2) remained smaller throughout life and weighed less than controls at the end of the research (Fig. 1). Both FBG (19.04 \pm 3.24 vs. $6.66 \pm 0.46 \mathrm{mmol} /$ $\mathrm{L}, t=-12.896, p<0.01)$, and $\mathrm{HbA}_{1 \mathrm{C}}(11.40 \pm 2.14 \%$ vs. $5.61 \pm 0.73 \%, t=-8.690, p<0.01)$, which measures the diabetic severity over time, increased in DM1 rats with statistical significance compared to non-diabetic controls. After treatment with GSPE, the serum AGEs decreased more in the DM2 group than in DM1 (0.041 \pm 0.028 vs. $0.100 \pm 0.056, t=2.792, p<0.05)$, while there was no statistical difference in their weights, $\mathrm{HbA}_{1 \mathrm{c}}$, or $\mathrm{FBG}(21.35 \pm 3.59$ vs. $19.04 \pm 3.24$ $\mathrm{mmol} / \mathrm{L}, t=1.494, p=0.153$ ) (Table 2).

Preventive and therapeutic effects of GSPE on diabetic cortex

1. Microstructural morphological changes of cerebral cortex. By H.E. staining, we found that the neurons of pyramidal cell layers in the cerebral cortex in STZ-

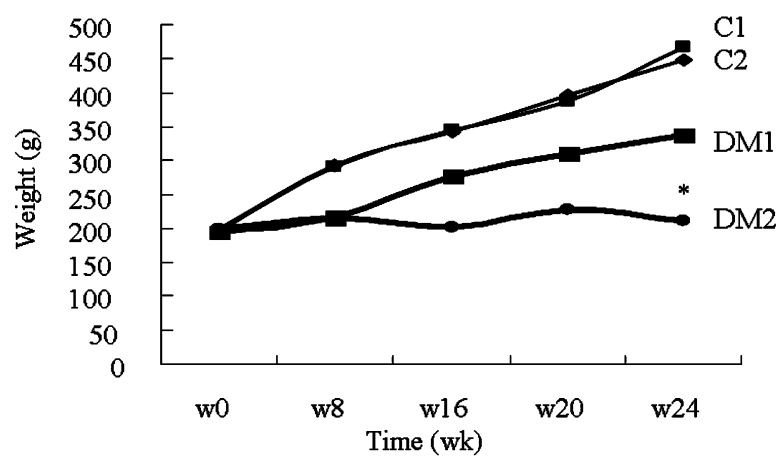

Fig. 1. Weight changes of experimental rats. After $24 \mathrm{wk}$, the weight of the DM1 group $(n=8)$ was less than that of non-diabetic controls $(n=10),{ }^{*} p<0.05$ by Student's $t$ test.

Table 2. Effects of GSPE on FBG, $\mathrm{HbA}_{1 c}$, and AGEs of experimental rats.

\begin{tabular}{lrccl}
\hline Grouping & $n$ & FBG $(\mathrm{mmol} / \mathrm{L})$ & $\mathrm{HbA}_{1 \mathrm{c}}(\%)$ & AGEs $(\mathrm{AU} / \mathrm{mg})$ \\
\hline C1 & 10 & $6.66 \pm 0.46^{*}$ & $5.61 \pm 0.73^{*}$ & $0.017 \pm 0.004^{*}$ \\
C2 & 10 & $7.00 \pm 0.90$ & $5.57 \pm 0.71$ & $0.026 \pm 0.026$ \\
DM1 & 8 & $21.35 \pm 3.59^{* *}$ & $11.40 \pm 2.14^{* *}$ & $0.100 \pm 0.0568^{* *}$ \\
DM2 & 12 & $19.04 \pm 3.24^{* *}$ & $11.41 \pm 2.14^{* \#}$ & $0.041 \pm 0.028^{* \#}$ \\
\hline
\end{tabular}

C1: untreated non-diabetic group; C2: GSPE-treated non-diabetic group; DM1: untreated diabetic group; DM2: GSPEtreated non-diabetic group; FBG: fasting blood sugar; $\mathrm{HbA}_{1 c}$ : glycolated hemoglobin; AGEs: advanced glycation end products.

${ }^{*} p<0.05,{ }^{* *} p<0.01$ vs $\mathrm{C} 1,{ }^{\#} p<0.05,{ }^{* \#} p<0.01$ vs DM1 by one-way ANOVA. 
A
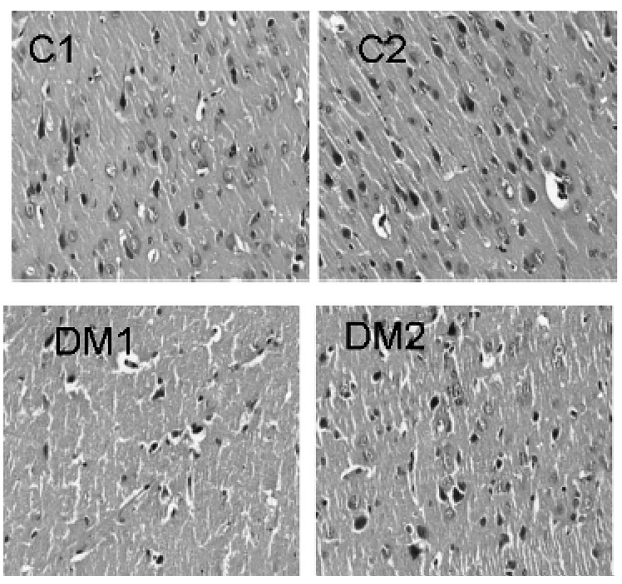

$\mathrm{C}$

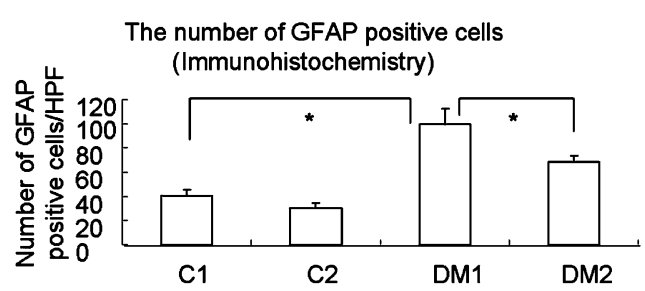

B
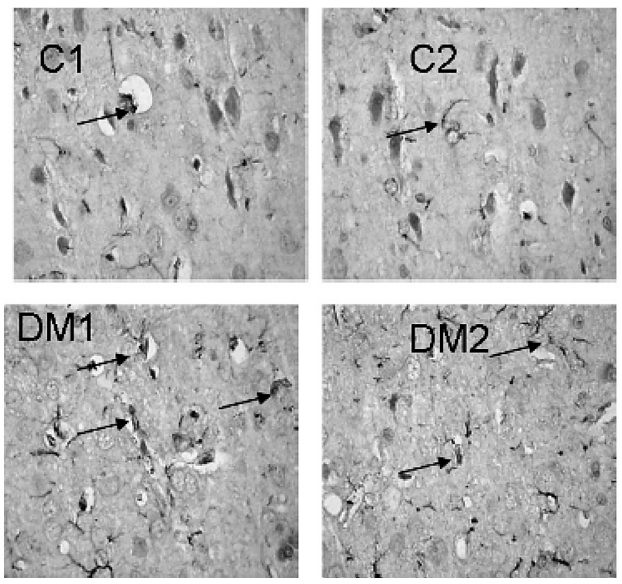

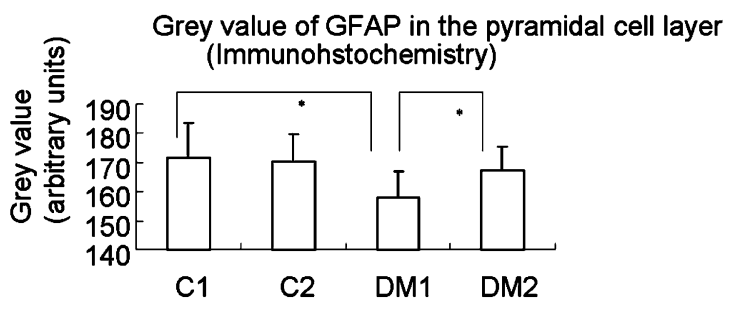

Fig. 2. Microstructure of the pyramidal cell layer. (A) The microscopic observations of cerebral cortex. After 24 wk of treatment, GSPE remarkably improved the cerebral degenerations in diabetic rats $(n=8)$ compared to controls $(n=10)$. H.E. staining, $\times 200$. (B) Immunohistochemistry for GFAP. Long-term hyperglycemia in rats caused increased GFAP positive neurons, which represents brain damage. $24 \mathrm{wk}$ of GSPE treatment reduced the GFAP positive neurons. Arrows indicate positive immunoreactivity. Immunohistochemical staining, $\times 400$. (C) The number of GFAP positive cells and corresponding grey value of astrocytes from 4 randomly selected pyramidal cell layers of cerebral cortical regions were determined. ${ }^{*} p<0.05$ by Student's $t$ test. Error bars, SD.

induced rats decreased (data not shown), with degenerative changes: the layers of neurons were not intact, the nuclei of neurons were shrunken and were in irregular order, and the plasma of neurons turned pink because of eosinophilic degeneration. After treatment with GSPE, the above changes were remarkably improved, and the number of neurons in the DM2 group increased compared to in the DM1 group (Fig. 2A).

Immunohistochemical studies were undertaken next to measure the intensity of GFAP expression. As an astrocyte-specific intermediate filament component, GFAP can be a biological marker of neurotoxicity. In this study, we found that the deposits of GFAP in the cerebral cortex of STZ-induced rats increased compared to nondiabetic controls, as demonstrated by the decreased grey value and increased positive cell numbers; after GSPE treatment, the deposits of GFAP in the cerebral cortex of STZ induced rats (DM2) decreased compared to in the DM1 group, as demonstrated by the increased grey value and the decreased positive cell numbers (Fig. 2B, C).

2. RAGE and NF- $\kappa B$ p65 expression in the cerebral cortex. By semi-quantitative RT-PCR, we found that the expression of RAGE mRNA in the cerebral cortex of STZ-induced rats increased compared to that in nondiabetic controls $(p<0.05)$; after GSPE treatment, the expression of RAGE mRNA in the pyramidal cell layer of STZ-induced rats decreased compared to that in the DM1 group $(p<0.05)$ (Fig. 3A). Similarly, the expression of NF- $\kappa \mathrm{B}$ p $65 \mathrm{mRNA}$ in the cerebral cortex of STZinduced rats increased compared to that in non-diabetic controls $(p=0.049)$; after treatment with GSPE, the expression of NF- $\kappa \mathrm{B}$ p 65 mRNA in the cerebral cortex of STZ-induced rats decreased compared to that in the DM1 group $(p=0.010)$ (Fig. 3B).

We also found that the protein expression of RAGE and NF- $\kappa \mathrm{B}$ p65 increased in diabetic rats, while GSPE treatment can lighten the protein increase caused by hyperglycemia. Immunohistochemically, we found the expression of RAGE and NF- $\kappa \mathrm{B}$ p 65 protein increased in the pyramidal cell layer of STZ-induced rats compared to that in non-diabetic controls, as demonstrated by the decreased grey value and the increased positive cell areas; after treatment with GSPE, the deposits of RAGE and NF- $\kappa \mathrm{B}$ p65 proteins decreased in the DM2 group compared to the DM1 group, as demonstrated by the increased grey value and the decreased positive cell areas (Fig. 3C, D). Western blot also revealed that $35 \mathrm{kD}$ protein of RAGE and $65 \mathrm{kD}$ protein of $\mathrm{NF}-\kappa \mathrm{B}$ p 65 expressed in the cerebral cortex of STZ-induced diabetic rats increased compared to that of the non-diabetic controls. After GSPE treatment, the expression of RAGE and 
A

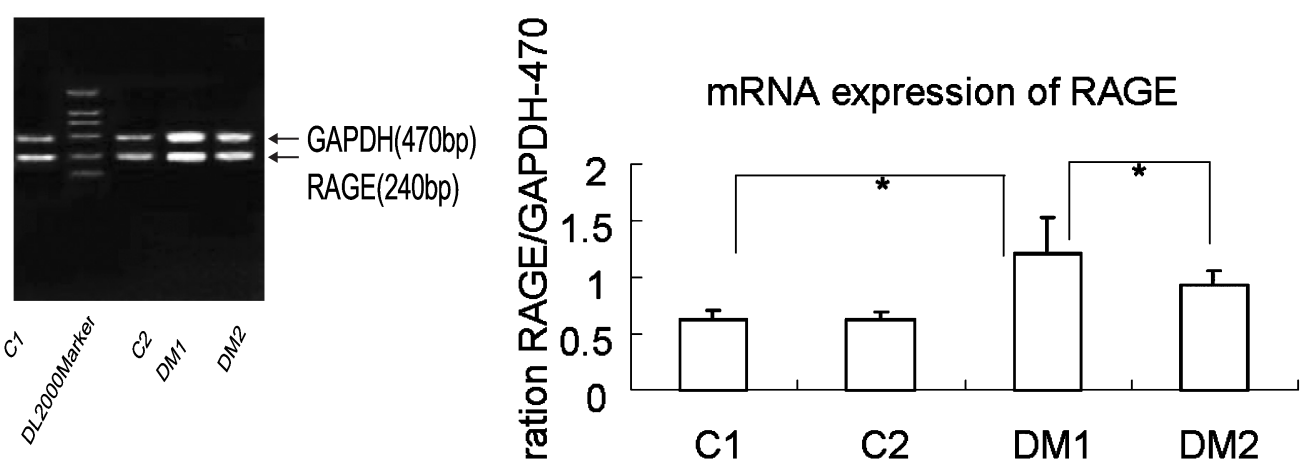

B
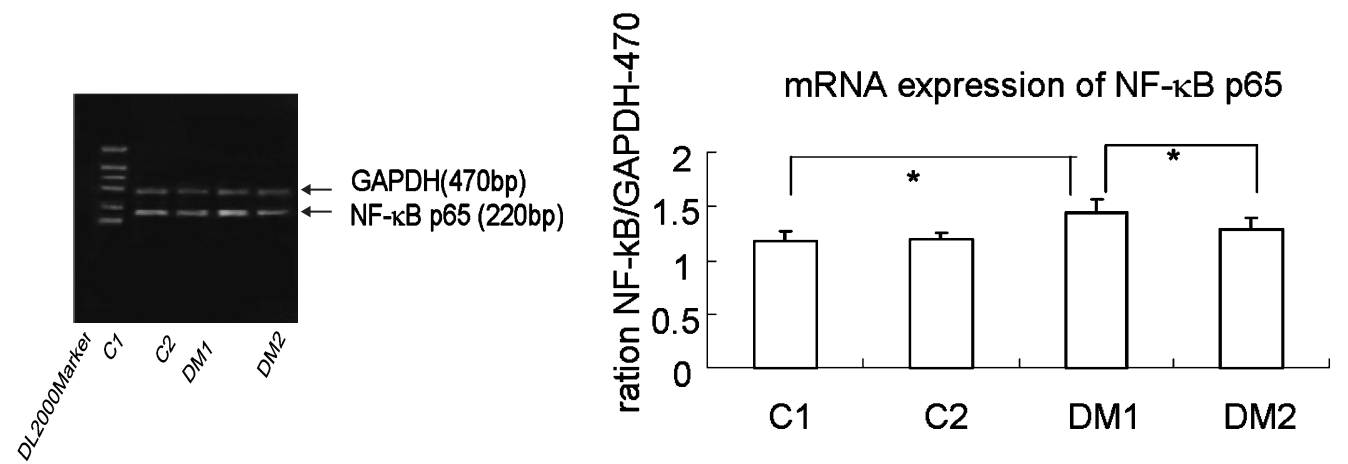

C
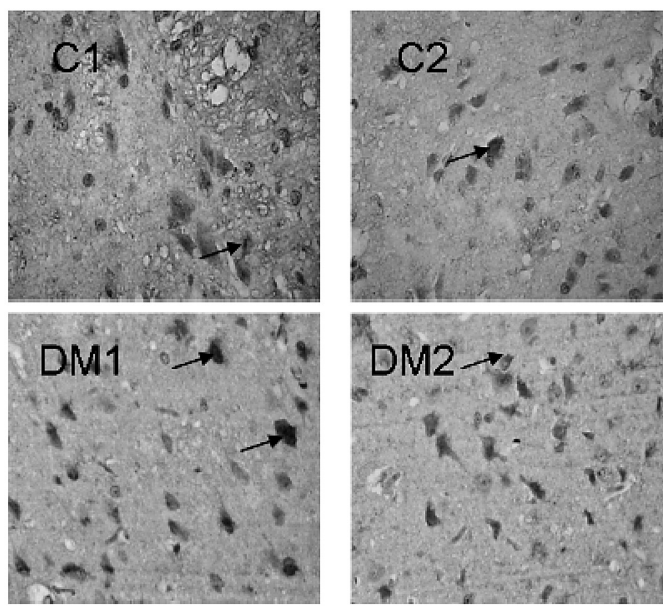

The area of RAGE positive cells (Immunochemistry)
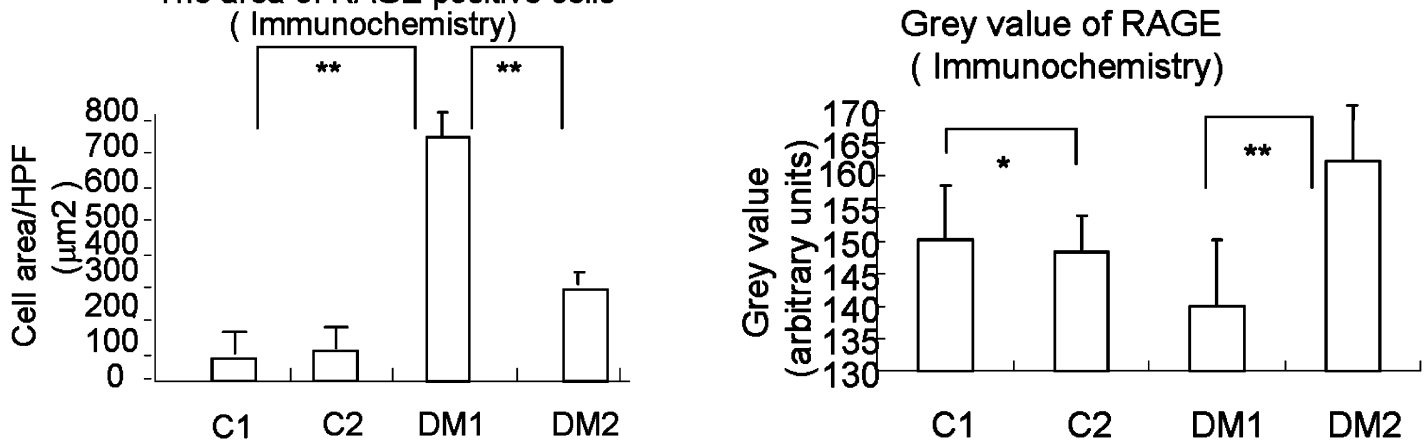

Fig. 3. A-C 
$\mathrm{D}$
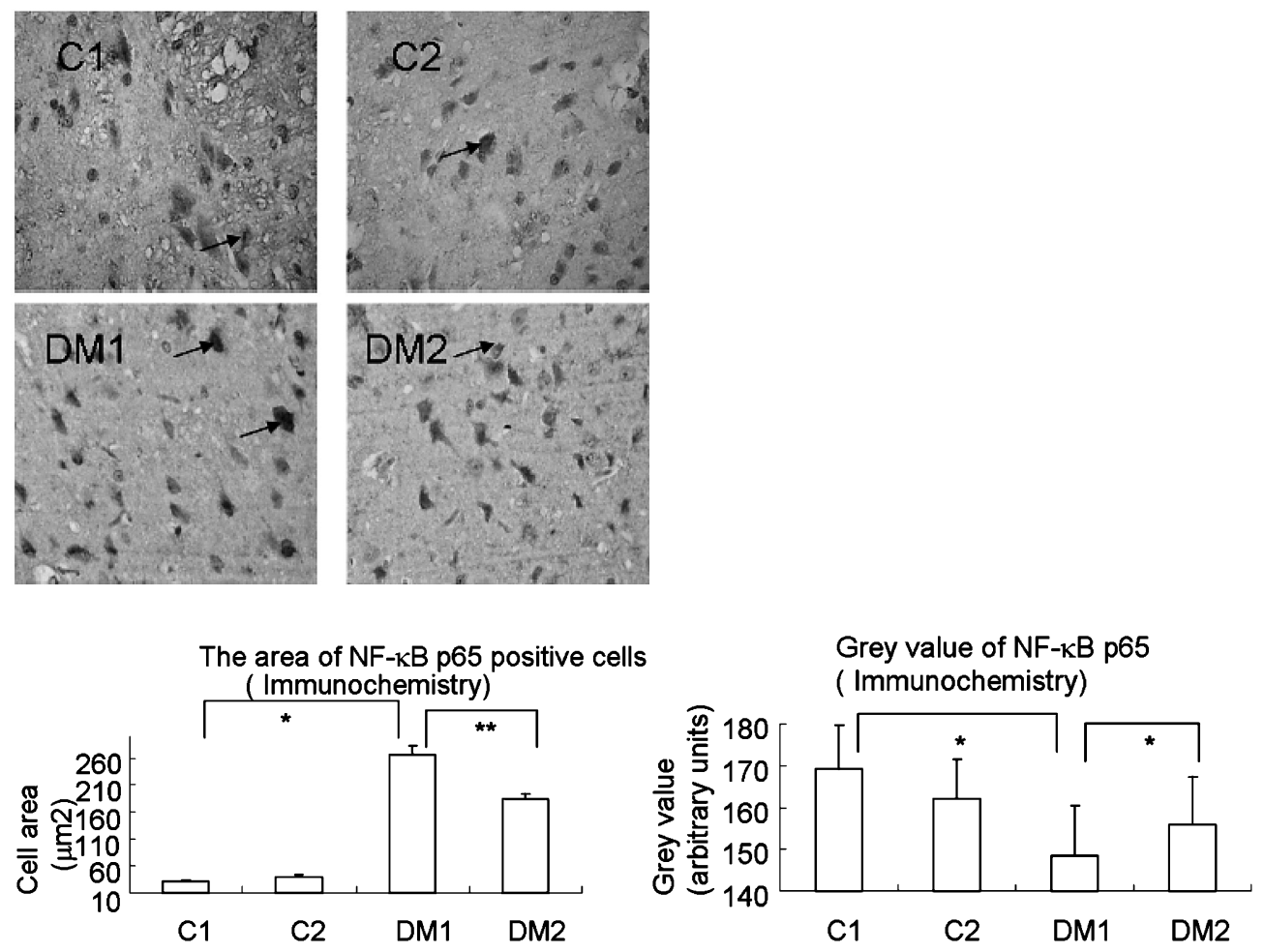

$\mathrm{E}$
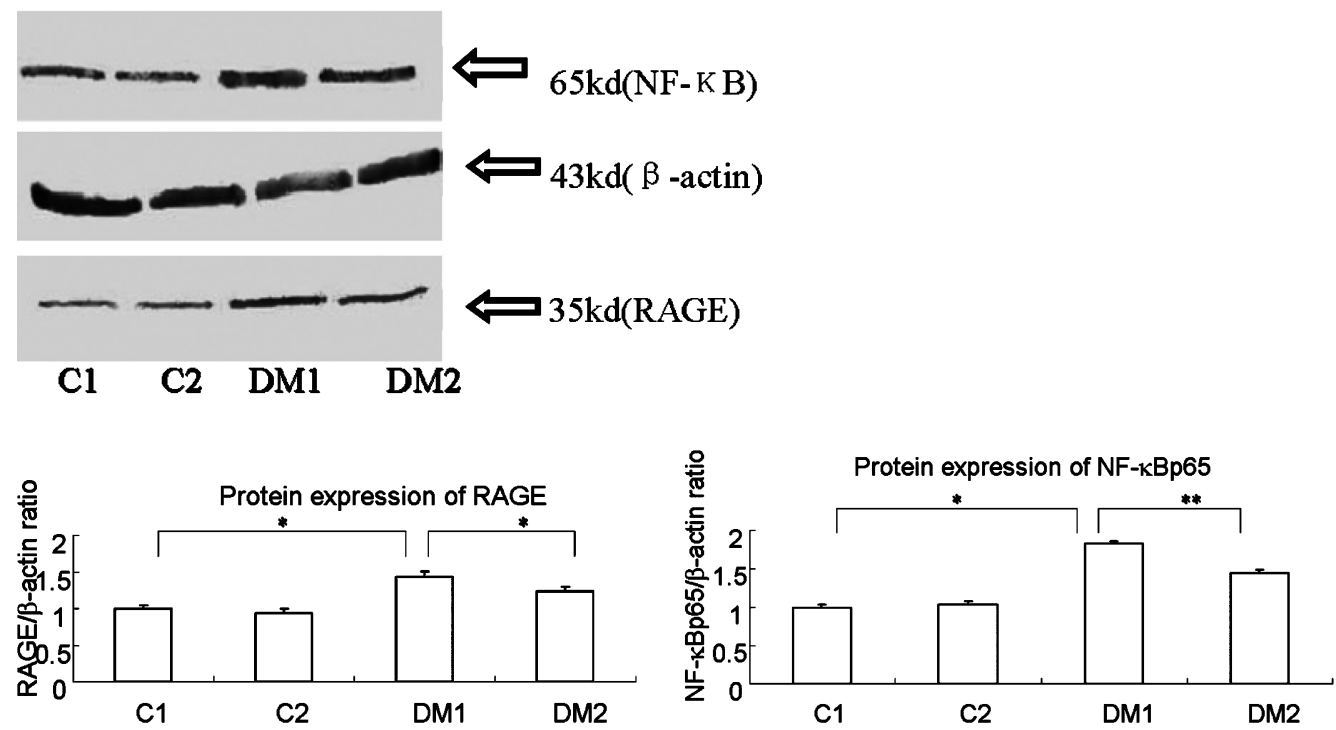

Fig. 3. GSPE treatment can alleviate the up-regulation of RAGE and NF- $\kappa \mathrm{B}$ p65 in mRNA and protein levels in the pyramidal cell layer of the cerebral cortex of diabetic rats. By RT-PCR, we found that the expression of RAGE (A) and NF- $\kappa$ B p65 mRNA (B) of DM1 rats $(n=8)$ increased compared to non-diabetic controls $(\mathrm{C} 1, n=10)$. After GSPE treatment, both the expression of RAGE and NF- $\kappa$ B p65 mRNA decreased in DM2 rats $(n=12)$ compared to DM1 rats. Similarly, by immunohistochemistry, the expression of RAGE (C) and NF- $\kappa$ B p65 protein (D) in diabetic rats increased compared to controls. After GSPE treatment, both the expression of RAGE and NF- $\kappa$ B p65 protein decreased in DM2 rats compared to DM1 animals. Arrows indicate positive immunoreactivity. Immunostained neurons in (C) and (D) were counted from 4 randomly selected cortical regions. Areas of RAGE and NF- $\kappa \mathrm{B}$ p 65 positive neurons were determined and were compared between DM1 and C1, and DM1 and DM2. The grey value of RAGE and NF- $\kappa$ B p6 5 staining was analyzed by Adobe Photoshop for comparison. Immunohistochemical staining, $\times 400$. (E) Western blot revealed that $35 \mathrm{kD}$ of RAGE and $65 \mathrm{kD}$ of $\mathrm{NF}-\kappa \mathrm{B}$ p65 protein in the pyramidal cell layer of diabetic rats increased compared to those of controls; after GSPE treatment, the expression of the two proteins decreased compared to DM1. ${ }^{*} p<0.05,{ }^{* *} p<0.01$ by Student's $t$ test. Error bars, SD. 
A
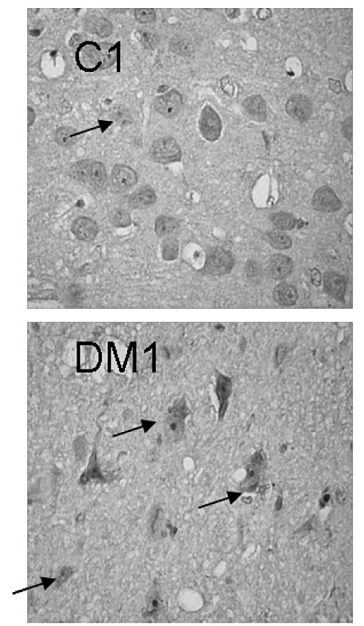
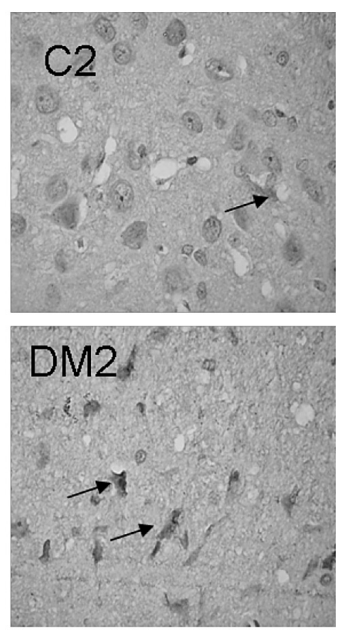

The area of VCAM- 1 positive cells (Immunochemistry)

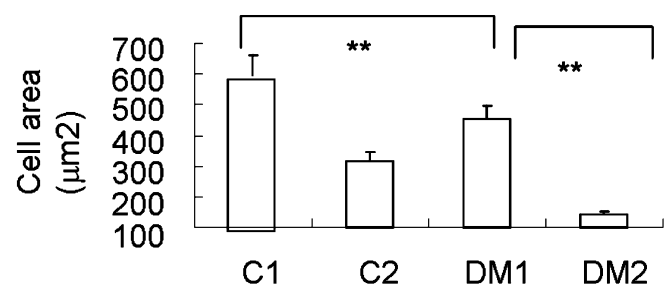

B
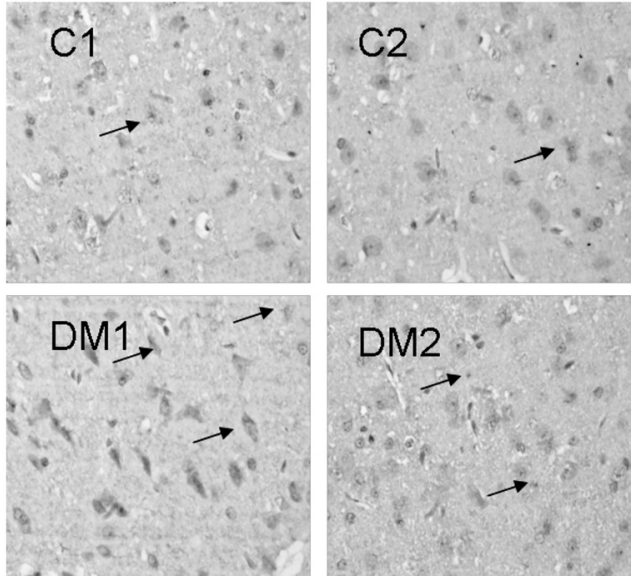

The area of ICAM- 1 positive cells (Immunochemistry)

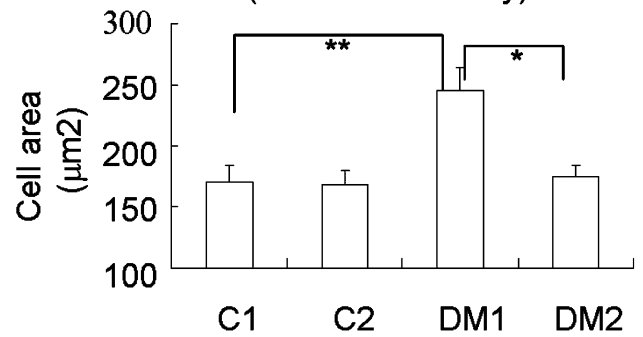

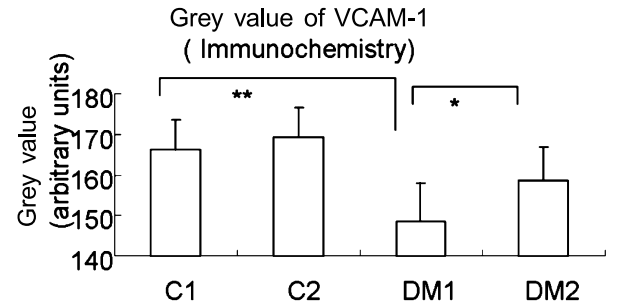

C1

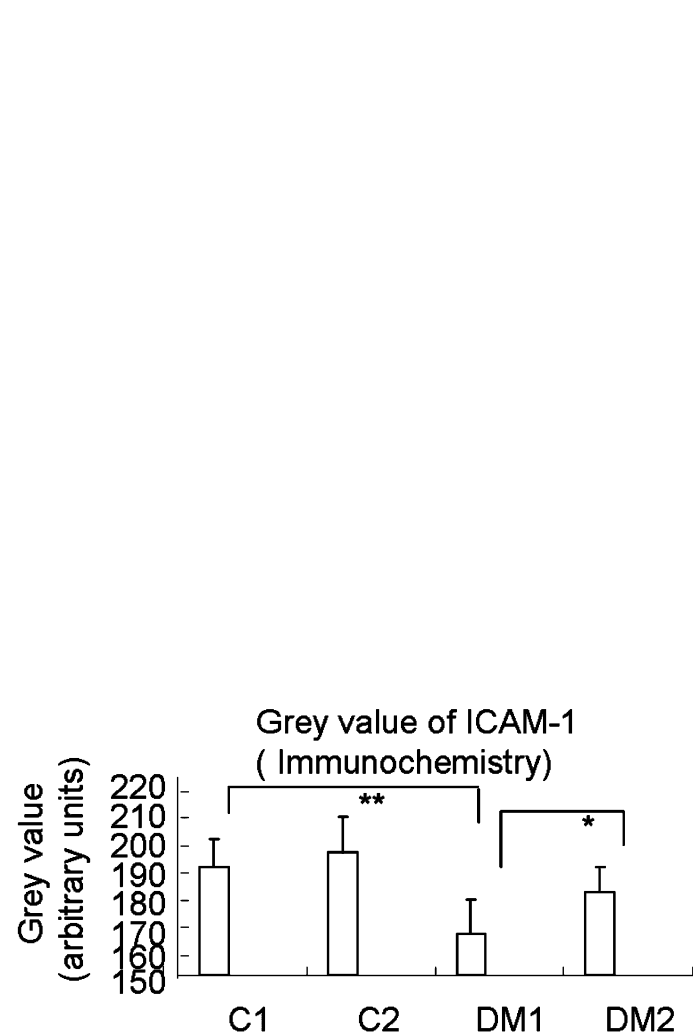

Fig. 4. GSPE treatment can lighten the up-regulation of VCAM-1 and ICAM-1 protein expression in the pyramidal cell layer of diabetic rats. By immunochemistry, the expression of VCAM-1 (A) and ICVM-1 (B) protein in diabetic rats increased compared to controls. After GSPE treatment, the protein expression decreased compared to DM1 animals. Arrows indicate positive immunoreactivity. Immunostained neurons in (A) and (B) were counted from 3 randomly selected pyramidal cell layer regions. Areas of VCAM-1 and ICVM-1 positive neurons were determined and were compared between DM1 and C1, and DM1 and DM2. The grey value of VCAM-1 and ICVM-1 staining was analyzed by Adobe Photoshop for comparison. ${ }^{*} p<0.05,{ }^{* *} p<0.01$ by Student's $t$ test. Error bars, SD. 
NF- $\kappa$ B p65 in DM2 decreased compared to that in DM1 (Fig. 3E), which was consistent with the results from immunochemistry and RT-PCR.

Taken together, our results here showed in addition to direct damage to neurons, long term poorly controlled blood sugar can cause the up-regulation of RAGE and NF- $\kappa$ B p 65 proteins, which can activate deleterious inflammatory cataracts.

3. VCAM-1 and ICAM-1 expression. Vascular cell adhesion molecule-1 (VCAM-1) and intercellular adhesion molecule-1 (ICAM-1) are adhesion molecules that can be activated by RAGE and NF- $\kappa$ B p65. Here, we wanted to see what happened to these downstream targets when RAGE and NF- $\kappa$ B p65 were up-regulated. As we expected, we found increased VCAM-1 and ICAM-1 deposits in the pyramidal cell layer of STZ-induced diabetic rats compared to that of non-diabetic controls, as manifested by the decreased grey value and the increased area of positive cells; GSPE treatment decreased VCAM-1 and ICAM-1 deposits in the pyramidal cell layer of DM2 rats compared to STZ-induced diabetic rats, as manifested by the increased grey value and the decreased area of positive cells (Fig. 4A, B).

Next, we correlated histological degenerations of the pyramidal cell layer in the cerebral cortex, which can be recognized using H.E. staining and GFAP immunostaining, with the up-regulation of RAGE, NF- $\kappa \mathrm{B}$ p 65 , and their downstream targets VCAM-1 and ICAM-1. So it is reasonable to conclude that the up-regulation of RAGE and NF- $\kappa$ B p 65 in diabetic rats can be associated with the increased degeneration in the cerebral cortex of poorly controlled diabetes.

\section{DISCUSSION}

Diabetes is a metabolic disorder with a globally rising prevalence which can affect the retina, kidney, muscles, blood vessels, peripheral and central neural system, and meanwhile, increases the risk of dementia in the elderly (23, 24). Although the chronic vascular complications are well described, the lesions in the central nervous system (CNS) have not been extensively investigated, and the concept of diabetic encephalopathy has remained controversial for more than $80 \mathrm{y}$ since the early 20th century when it was first described $(1,24$ 26). Other terms, such as functional cerebral impairment, central neuropathy and diabetic-associated cognitive decline (DACD) have been used to document the disorder (1). The two types of diabetic encephalopathy, primary and secondary, have also been discussed (25). Although cognitive deficits vary from mild to moderate, the truth is that they are generally modest in young adults and tend to be more pronounced in the elderly, where they significantly impair their daily functioning and burden the society $(1,27)$.

Diffused and local degenerative changes in the cerebral cortex, neuronal loss, demyelization, gliosis, and infarction secondary to microangiopathy have been described as structural changes in type 1 diabetic patients. Magnetic resonance imaging (MRI) and computed tomography (CT) have demonstrated that indices of cerebral atrophy, i.e. widened sulci and/or enlarged lateral ventricles, and the occurrence of white matter hyperintensities, are more pronounced in diabetic patients than in image-matched control subjects, which reflects a process of accelerated aging (25, 28-30). To date, the complication, known as 'diabetic encephalopathy,' has been regarded as a cerebral neurodegenerative disorder in patients with diabetes, especially poorly controlled diabetes (30).

Glial fibrillary acidic protein (GFAP), an astrocytespecific intermediate filament component, can be a biological marker of neurotoxicity. Evidence has shown that increased GFAP expression is closely related to cerebral abnormalities identified by H.E. staining. In our present study, we found proliferation of astrocytes in the cerebral cortex of diabetic rats, and in the meantime, we observed the suppression of GSPE to this proliferation.

AGEs, which are a regarded as a functional indicator of glucose concentration and time, accumulate in diverse settings, such as diabetes, inflammation, renal failure, pro-oxidant states and natural aging $(2,27$, 31). The reaction between free reducing sugars and free amino groups of proteins can initiate a posttranslational modification process known as nonenzymatic glycosylation, which increases in diabetes accompanied by poorly controlled hyperglycemia. The formation of AGEs may affect neuronal function through various mechanisms, including modification of functionally important proteins like tubulin, $\mathrm{Na}^{+}, \mathrm{K}^{+}$-ATPase, increased production of reactive oxygen species (ROS), affecting of vascular function, and quenching of the vasodilating compound nitric oxide (32). RAGE, the receptor for AGEs, is an integral membrane protein and a multi-ligand member of the immunoglobulin superfamily. RAGE can be upregulated under diverse pathological conditions $(5,7,8)$. The binding of AGEs to their receptor (RAGE) can activate the proinflammatory transcription factor NF- $\kappa \mathrm{B}$ p 65 , which allows its translocation to the nucleus, where it regulates the expression of a large number of cytokine genes, including tumor necrosis factor- $\alpha$ (TNF- $\alpha$ ), interleukin- $1 \beta$ (IL- $1 \beta$ ) and RAGE itself. The process seems to be responsible for maintaining acute and chronic inflammation in various cell types $(31,33-35)$. A series of studies of sural nerve biopsies by Haslbeck et al. suggest that the RAGE pathway plays a critical proinflammatory role in vasculitic neuropathy and may be one of the first steps in the pathogenesis of IGT-related polyneuropathy (PNP) even before chronic hyperglycemia occurs $(9,33,34)$.

Consistent with these previous research results, we showed that long-term hyperglycemia can cause not only increased serum AGEs but also the upregualtion of RAGE and NF- $\kappa$ B p 65 both at mRNA and protein levels in the cerebral cortex in STZ-induced diabetic rats. Our results lead us to draw the conclusion that the AGEs/ RAGE/NF- $\kappa$ B pathway may be an indispensable and initiating factor in the pathogenesis of diabetic encephalopathy.

Studies have showed us that a moderately low intake of polyphenol-enriched preparations would be effective 
in up-regulating the antioxidant defense mechanism in most body tissues, including the cerebral cortex, cerebellum, and hippocampus in the adult rat brain, which may suggest that proanthocyanidin is a potent enhancer of cognition in older rats (36). Many studies have provided evidence that GSPE has potent radical scavenging ability, antioxidant properties, and significant neuroprotective as well as cardiovascular protective effects. It has been proven that GSPE can interfere with chronic diabetic complications by affecting the AGEs/RAGE/NF- $\kappa$ B pathway $(14-17,21,37)$.

Our previous studies have shown that GSPE inhibited the cell inflammatory factor expression and protected the function of endothelial cells through activation of PPAR gamma expression and inhibition of RAGE expression, GSPE also inhibited the enhanced VCAM-1 expression in AGE-exposed endothelial cells by suppressing ROS generation $(21,37)$. Consistent with these findings, in our present study, we showed that as compared to diabetic controls, not only the serum levels of AGEs decreased, but also the expression of RAGE and NF- $\kappa$ B p 65 diminished at both mRNA and protein levels after GSPE treatment. We also showed immunochemically that GSPE could down-regulate the expression of NF- $\kappa$ B p65-activated inflammatory cytokine VCAM-1 and ICAM-1. All together, our results disclosed the therapeutic potential of GSPE in the prevention and treatment of diabetic encephalopathy.

Drawing assistance from its proanthocyanidin content, daily consumption of $250 \mathrm{mg} / \mathrm{mL}$ GSPE in the form of food or dietary supplementation may offer a prophylactic measure against the onset and progression of oxidative cataract, and the therapeutic range of plasma GSPE concentrations did not cause any abnormality of liver or kidney, or other serious side effects (38). However, high dosage of GSPE $(500 \mathrm{mg} / \mathrm{mL})$ can induce cytotoxicity that is associated with increased apoptotic cell death, so the dosages of GSPE should be optimized to avoid potential harmful pro-oxidant effects (38-40).

In conclusion, long-term hyperglycemia in STZinduced diabetic rats can cause the up-regulation of AGEs/RAGE/NF- $\kappa$ B, which may play vital roles in the pathogenesis of diabetic encephalopathy. Daily intake of $250 \mathrm{mg} / \mathrm{kg}$ body weight GSPE could improve degenerative changes of the cerebral cortex through down-regulating the AGEs/RAGE/NF- $\kappa$ B pathway. Our study confirmed us that RAGE is a promising target for diabetic complications, while GSPE, acting as a modulator of RAGE, could be a prophylactic and therapeutic agent against diabetic encephalopathy.

\section{Acknowledgments}

We give our thanks to the Department of Experimental Pathology and Physiology, Shandong Academy of Medical Science and the Center for New Drug Evaluation, Shandong University. And we also sincerely thank Dr. Xiulian Sun from Shandong University for her kind revising of this manuscript.

\section{REFERENCE}

1) Mijnhout GS, Scheltens P, Diamant M. 2006. Diabetic encephalopathy: A concept in need of a definition. Diabetologia 49: 1447-1448.

2) Aragno M, Mastrocola R, Medana C. 2005. Up-regulation of advanced glycated products receptors in the brain of diabetic rats is prevented by antioxidant treatment. Endocrinology 146: 5561-5567.

3) Bierhaus A, Humpert PM, Morcos M. 2005. Understanding RAGE, the receptor for advanced glycation end products. J Mol Med 83: 876-886.

4) van den Berg E, Kessels RP, Kappelle LJ. 2006. Type 2 diabetes, cognitive function and dementia: vascular and metabolic determinants. Drugs Today 42: 741-754.

5) Katz J, Bhattacharyya I, Farkhondeh-Kish F. 2005. Expression of the receptor of advanced glycation end products in gingival tissues of type 2 diabetes patients with chronic periodontal disease: a study utilizing immunohistochemistry and RT-PCR. J Clin Periodontol 32: $40-44$.

6) Ziyadeh FN, Cohen MP, Guo J, Jin Y. 1997. RAGE mRNA expression in the diabetic mouse kidney. Mol Cell Biochem 170: 147-152.

7) Park IH, Yeon SI, Youn JH. 2004. Expression of a novel secreted splice variant of the receptor for advanced glycation end products (RAGE) in human brain astrocytes and peripheral blood mononuclear cells. Mol Immunol 40: 1203-1211.

8) Schmidt AM, Yan SD, Yan SF, Stern DM. 2000. The biology of the receptor for advanced glycation end products and its ligands. Biochim Biophys Acta 1498: 99-111.

9) Haslbeck KM, Schleicher E, Bierhaus A. 2005. The AGE/RAGE/NF-(kappa)B pathway may contribute to the pathogenesis of polyneuropathy in impaired glucose tolerance (IGT). Exp Clin Endocrinol Diabetes 113: 288291.

10) Sasaki N, Toki S, Chowei H. 2001. Immunohistochemical distribution of the receptor for advanced glycation end products in neurons and astrocytes in Alzheimer's disease. Brain Res 888: 256-262.

11) Ramasamy R, Vannucci SJ, Yan SS. 2005. Advanced glycation end products and RAGE: a common thread in aging, diabetes, neurodegeneration, and inflammation. Glycobiology 15: 16R-28R.

12) Liu YN, Shen XN, Yao GY. 2006. Effects of grape seed proanthocyanidins extracts on experimental diabetic nephropathy in rats. Wei Sheng Yan Jiu 35: 703-705.

13) Yamakoshi J, Saito M, Kataoka S. 2002. Safety evaluation of proanthocyanidin-rich extract from grape seeds. Food Chem Toxicol 40: 599-607.

14) Pataki T, Bak I, Kovacs P. 2002. Grape seed proanthocyanidins improved cardiac recovery during reperfusion after ischemia in isolated rat hearts. Am J Clin Nutr 75: 894-899.

15) Sano T, Oda E, Yamashita T. 2005. Anti-thrombotic effect of proanthocyanidin, a purified ingredient of grape seed. Thromb Res 115: 115-121.

16) $\mathrm{Hu} \mathrm{M}$, McClements DJ, Decker EA. 2004. Antioxidant activity of a proanthocyanidin-rich extract from grape seed in whey protein isolate stabilized algae oil-in-water emulsions. J Agric Food Chem 52: 5272-5276.

17) Natella F, Belelli F, Gentili V. 2002. Grape seed proanthocyanidins prevent plasma postprandial oxidative stress in humans. J Agric Food Chem 50: 7720-7725. 
18) Kim H, Deshane J, Barnes S, Meleth S. 2006. Proteomics analysis of the actions of grape seed extract in rat brain: technological and biological implications for the study of the actions of psychoactive compounds. Life Sci $\mathbf{7 8}$ : 2060-2065.

19) Shao ZH, Becker LB, Vanden Hoek TL. 2003. Grape seed proanthocyanidin extract attenuates oxidant injury in cardiomyocytes. Pharmacol Res 47: 463-469.

20) Pinent M, Blay M, Blade MC. 2004. Grape seed-derived procyanidins have an antihyperglycemic effect in streptozotocin-induced diabetic rats and insulinomimetic activity in insulin-sensitive cell lines. Endocrinology 145: 4985-4990.

21) Zhang FL, Gao HQ, Wu JM. 2006. Selective inhibition by grape seed proanthocyanidin extracts of cell adhesion molecule expression induced by advanced glycation end products in endothelial cells. J Cardiovasc Pharm 48 : 47-53.

22) Xu L, Li B, Cheng M. 2008. Oral administration of grape seed proanthocyanidin extracts downregulate RAGE dependant nuclear factor-kappa B p65 expression in the hippocampus of streptozotocin induced diabetic rats. Exp Clin Endocrinol Diabetes 116: 215-224.

23) Biessels GJ, Kerssen A, de Haan EH, Kappelle LJ. 2007. Cognitive dysfunction and diabetes: implications for primary care. Primary Care Diabetes 1: 187-193.

24) van den Berg E, Kessels RP, Kappelle LJ. 2007. Type 2 diabetes, cognitive function and dementia: vascular and metabolic determinants. TTMed 11: E7.

25) Sima AA, Kamiya H, Li ZG. 2004. Insulin, C-peptide, hyperglycemia, and central nervous system complications in diabetes. Eur J Pharmacol 490: 187-197.

26) Brands AM, Henselmans JM, de Haan EH, Biessels GJ. 2003. Diabetic encephalopathy: an underexposed complication of diabetes mellitus. Ned Tijdschr Geneeskd 147: 11-14.

27) Akisaki T, Sakurai T, Takata T. 2006. Cognitive dysfunction associates with white matter hyperintensities and subcortical atrophy on magnetic resonance imaging of the elderly diabetes mellitus Japanese elderly diabetes intervention trial (J-EDIT). Diabetes Metab Res Rev 22: 376-384.

28) Sima AA. 2006. Pathological mechanisms involved in diabetic neuropathy: can we slow the process? Curr Opin
Invest Drugs 7: 324-337.

29) Suzuki Y, Hata T, Miyaoka H. 1996. Diabetes with the 3243 mitochondrial tRNALeu(UUR) mutation. Diabetes Care 19: 739-743.

30) Korf ES, White LR, Scheltens P, Launer LJ. 2006. Brain aging in very old men with type 2 diabetes: the Honolulu-Asia Aging Study. Diabetes Care 29: 2268-2274.

31) Wada R, Yagihashi S. 2005. Role of advanced glycation end products and their receptors in development of diabetic neuropathy. Ann NY Acad Sci 1043: 598-604.

32) Kouta Y, Sakurai T, Yokono K. 2006. Cognitive dysfunction and dementia associated with elderly diabetes. Nippon Rinsho 64: 119-123.

33) Haslbeck KM, Neundorfer B, Schlotzer-Schrehardtt U. 2007. Activation of the RAGE pathway: a general mechanism in the pathogenesis of polyneuropathies? Neurol Res 29: 103-110.

34) Haslbeck KM, Bierhaus A, Erwin S. 2004. Receptor for advanced glycation endproduct (RAGE)-mediated nuclear factor-kappaB activation in vasculitic neuropathy. Muscle Nerve 29: 853-860.

35) Alves M, Cunha DA, Calegari VC. 2005. Nuclear factorkappaB and advanced glycation end-products expression in lacrimal glands of aging rats. J Endocrinol 187: 159-166.

36) Devi A, Jolitha AB, Ishii N. 2006. Grape seed proanthocyanidin extract (GSPE) and antioxidant defense in the brain of adult rats. Med Sci Monit 12: BR124-129.

37) Ma L, Gao HQ, Li BY. 2007. Grape seed proanthocyanidin extracts inhibit vascular cell adhesion molecule expression induced by advanced glycation end products through activation of peroxisome proliferators-activated receptor gamma. J Cardiovasc Pharm 49: 293-298.

38) Durukan AH, Evereklioglu C, Hurmeric V. 2006. Ingestion of IH636 grape seed proanthocyanidin extract to prevent selenite-induced oxidative stress in experimental cataract. J Cataract Refr Surg 32: 1041-1045.

39) Shao ZH, Hsu CW, Chang WT. 2006. Cytotoxicity induced by grape seed proanthocyanidins: role of nitric oxide. Cell Biol Toxicol 22: 149-158.

40) Shao ZH, Vanden Hoek TL, Xie J. 2003. Grape seed proanthocyanidins induce pro-oxidant toxicity in cardiomyocytes. Cardiovasc Toxicol 3: 331-339. 